\title{
Many Density Functional Theory Approaches Fail to Give Reliable Large Hydrocarbon Isomer Energy Differences
}

\author{
Peter R. Schreiner, ${ }^{* a}$ Andrey A. Fokin, ${ }^{\text {a }}$ Robert A. Pascal, Jr. \\ and Armin de Meijere \\ ${ }^{a}$ Institute of Organic Chemistry, Justus-Liebig University Giessen, Heinrich-Buff-Ring 58, \\ 35392 Giessen, Germany and ${ }^{b}$ Princeton University, Department of Chemistry, Princeton, New \\ Jersey 08544, USA, and ${ }^{c}$ Institute of Organic and Biomolecular Chemistry, Georg-August \\ University Göttingen, Tammannstr. 2, 37077 Göttingen, Germany \\ prs@org.chemie.uni-giessen.de, andrey.fokin@org.chemie.uni-giessen.de
}

\section{Supplementary Information}

Table S1. Computational data for $(\mathrm{CH})_{12}$ isomers at the B3LYP/6-31G* level.

\begin{tabular}{|c|c|c|c|c|c|}
\hline $\begin{array}{l}\text { Cmpd } \\
\#\end{array}$ & $\begin{array}{l}\mathrm{PG}, E \\
(\mathrm{au})\end{array}$ & $\begin{array}{l}\text { Z.P.E. } \\
(\mathrm{au})\end{array}$ & $\begin{array}{c}E+\text { Z.P.E. } \\
(\mathrm{au})\end{array}$ & $\begin{array}{c}\Delta(E+\text { Z.P.E. }) \\
(\mathrm{kcal} / \mathrm{mol})\end{array}$ & $\begin{array}{l}\Delta H_{\mathrm{f}}(\mathrm{AM} 1) \\
(\mathrm{kcal} / \mathrm{mol})\end{array}$ \\
\hline & & & Saturated & & \\
\hline 1 & $D_{3 d}-464.426437$ & 0.209170 & -464.217267 & 0.0 & 81.4 \\
\hline 2 & $C_{2 v}-464.407769$ & 0.208912 & -464.198857 & +11.6 & 87.1 \\
\hline 3 & $T_{d}-464.384724$ & 0.208694 & -464.176031 & +25.9 & 104.5 \\
\hline & & & Monoenes & & \\
\hline 4 & $C_{S}-464.397647$ & 0.207684 & -464.189963 & +17.1 & 95.7 \\
\hline 5 & $C_{2 v}-464.393185$ & 0.208024 & -464.185161 & +20.1 & 87.9 \\
\hline & & & Dienes & & \\
\hline 6 & $C_{S}-464.410451$ & 0.207243 & -464.203208 & +8.8 & 79.2 \\
\hline 7 & $C_{1}-464.415121$ & 0.207581 & -464.207540 & +6.1 & 82.1 \\
\hline 8 & $C_{1}-464.391732$ & 0.207060 & -464.184672 & +20.5 & 104.8 \\
\hline 9 & $C_{2 v}-464.407318$ & 0.207405 & -464.199913 & +10.9 & 104.0 \\
\hline & & & Trienes & & \\
\hline 10 & $C_{3 v}-464.405771$ & 0.206778 & -464.198992 & +11.5 & 73.1 \\
\hline 11 & $C_{S}-464.410668$ & 0.206111 & -464.204557 & +8.0 & 72.7 \\
\hline 12 & $C_{S}-464.411607$ & 0.206006 & -464.205601 & +7.3 & 77.4 \\
\hline 13 & $C_{1}-464.399927$ & 0.206302 & -464.193625 & +14.8 & 82.2 \\
\hline 14 & $C_{1}-464.407946$ & 0.206313 & -464.201633 & +9.8 & 82.9 \\
\hline 15 & $C_{1}-464.410018$ & 0.206566 & -464.203452 & +8.7 & 85.2 \\
\hline 16 & $C_{2 v}-464.400990$ & 0.206556 & -464.194434 & +14.3 & 87.9 \\
\hline 17 & $C_{1}-464.402409$ & 0.204969 & -464.197440 & +12.4 & 86.5 \\
\hline 18 & $C_{1}-464.399348$ & 0.205055 & -464.194292 & +14.4 & 88.9 \\
\hline
\end{tabular}


Continuation of Table S1.

\begin{tabular}{|c|c|c|c|c|c|}
\hline \multicolumn{6}{|c|}{ Tetraenes } \\
\hline 19 & $D_{2 d}-464.359516$ & 0.204924 & -464.154592 & +39.3 & 89.2 \\
\hline 20 & $C_{1}-464.409934$ & 0.204662 & -464.205273 & +7.5 & 75.3 \\
\hline 21 & $C_{S}-464.385111$ & 0.204576 & -464.180535 & +23.0 & 99.8 \\
\hline 22 & $C_{1}-464.414695$ & 0.204645 & -464.210042 & +4.5 & 73.5 \\
\hline 23 & $C_{S}-464.388217$ & 0.205067 & -464.183150 & +21.4 & 96.1 \\
\hline 24 & $C_{1}-464.409394$ & 0.205389 & -464.204005 & +8.3 & 77.5 \\
\hline 25 & $C_{S}-464.401708$ & 0.205639 & -464.196070 & +13.3 & 74.7 \\
\hline 26 & $D_{2 h}-464.372331$ & 0.204170 & -464.168161 & +30.8 & 82.1 \\
\hline 27 & $C_{2}-464.406330$ & 0.204135 & -464.202195 & +9.5 & 70.8 \\
\hline 28 & $C_{2}-464.401183$ & 0.204225 & -464.196958 & +12.7 & 73.9 \\
\hline \multicolumn{6}{|c|}{ Pentaenes } \\
\hline 29 & $C_{2}-464.370676$ & 0.204011 & -464.166665 & +31.8 & 93.9 \\
\hline 30 & $C_{i}-464.391051$ & 0.200385 & -464.190666 & +16.7 & 99.9 \\
\hline 31 & $C_{S}-464.407696$ & 0.201968 & -464.205727 & +7.2 & 85.4 \\
\hline 32 & $C_{2}-464.377265$ & 0.202539 & -464.174727 & +26.7 & 86.4 \\
\hline 33 & $C_{S}-464.380481$ & 0.202884 & -464.177597 & +24.9 & 84.4 \\
\hline 34 & $C_{1}-464.395375$ & 0.203393 & -464.191982 & +15.9 & 82.7 \\
\hline 35 & $C_{1}-464.400408$ & 0.203222 & -464.197185 & +12.6 & 78.1 \\
\hline \multicolumn{6}{|c|}{ Hexaenes } \\
\hline 36 & $D_{3 d}-464.352857$ & 0.197774 & -464.155083 & +39.0 & 96.0 \\
\hline 37 & $C_{2}-464.372002$ & 0.200785 & -464.171216 & +28.9 & 100.2 \\
\hline 38 & $C_{S}-464.372251$ & 0.200216 & -464.172035 & +28.4 & 96.7 \\
\hline benzene $\mathrm{x}$ & x $2-464.497322$ & 0.201462 & -464.295860 & -49.3 & 44.0 \\
\hline
\end{tabular}


Table S2. Absolute and relative energies of selected $(\mathrm{CH})_{12}$ hydrocarbons at B3LYP and MP2 levels of theory with ZPVe corrections included.

\begin{tabular}{|c|c|c|c|c|c|c|c|c|}
\hline Structure & $\mathrm{PG}$ & $\begin{array}{c}\text { B3LYP/6-311 } \\
++\mathrm{G}(2 \mathrm{~d}, \mathrm{p}) \\
\Delta(\mathrm{E}+\mathrm{ZPVE}) \\
\mathrm{kcal} / \mathrm{mol}\end{array}$ & $\begin{array}{c}\text { B3PW91/6-31 } \\
1++\mathrm{G}(2 \mathrm{~d}, \mathrm{p}) \\
\Delta(\mathrm{E}+\mathrm{ZPVE}) \\
\mathrm{kcal} / \mathrm{mol}\end{array}$ & $\begin{array}{c}\Delta \Delta \mathrm{E}_{\mathrm{B} 3 \mathrm{PW}} \\
\text { 91-B3LYP } \\
\mathrm{kcal} / \mathrm{mol}\end{array}$ & $\begin{array}{c}\mathrm{MP} 2 / 6-31 \mathrm{G} \\
* * \\
-\mathrm{au}\end{array}$ & $\begin{array}{c}\mathrm{MP} 2 / 6-31 \mathrm{G}^{*} \\
* \\
\Delta(\mathrm{E}+\mathrm{ZPVE}) \\
\mathrm{kcal} / \mathrm{mol}\end{array}$ & $\begin{array}{c}\mathrm{MP} 2 / \mathrm{cc}-\mathrm{pV} \\
\mathrm{DZ} \\
-\mathrm{au}\end{array}$ & $\begin{array}{c}\mathrm{MP} 2 / \mathrm{cc}-\mathrm{pV} \\
\mathrm{DZ} \Delta(\mathrm{E}+ \\
\mathrm{ZPVE}) \\
\mathrm{kcal} / \mathrm{mol}\end{array}$ \\
\hline & $\mathrm{D}_{3 \mathrm{~d}}$ & 0.0 & 0.0 & 0.0 & 462.780143 & 0.0 & 462.783364 & 0.0 \\
\hline & $\mathrm{C}_{2 \mathrm{v}}$ & +11.4 & +12.2 & 0.8 & 462.756528 & +14.8 & 462.758934 & +15.3 \\
\hline & Cs & +4.4 & +12.8 & 8.4 & 462.756834 & +14.6 & 462.760307 & +14.5 \\
\hline & $\mathrm{C}_{1}$ & +1.6 & +9.7 & 8.1 & 462.761479 & +11.7 & 462.763834 & +12.2 \\
\hline & $\mathrm{C}_{2 \mathrm{v}}$ & +6.6 & +13.0 & 6.4 & 462.756591 & +14.8 & 462.764953 & +11.5 \\
\hline & $\mathrm{C}_{\mathrm{s}}$ & +1.3 & +14.9 & 13.6 & 462.742198 & +23.8 & 462.746388 & +23.2 \\
\hline & $\mathrm{C}_{\mathrm{s}}$ & -0.1 & +13.9 & 14.0 & 462.743712 & +22.9 & 462.746370 & +23.2 \\
\hline & $\mathrm{C}_{1}$ & +2.2 & +14.1 & 11.9 & 462.752078 & +17.6 & 462.757219 & +16.4 \\
\hline & $\mathrm{C}_{1}$ & -1.9 & +16.0 & 17.9 & 462.739284 & +25.6 & 462.744187 & +24.6 \\
\hline & $\mathrm{C}_{1}$ & -4.7 & +12.6 & 17.3 & 462.743453 & +23.0 & 462.748521 & +21.9 \\
\hline & $\mathrm{C}_{1}$ & -0.4 & +16.8 & 17.2 & 462.741373 & +24.3 & 462.746757 & +23.0 \\
\hline & $\mathrm{C}_{\mathrm{s}}$ & -4.5 & +17.2 & 21.7 & 462.732148 & +30.1 & 462.737842 & +28.6 \\
\hline
\end{tabular}


Table S3. Absolute energies (E,-au), of octahedrane (1) and hydrocarbons 22 and $\mathbf{3 1}$ at various $a b$ initio levels of theory.

\begin{tabular}{|c|c|c|c|c|c|c|}
\hline Structure & $\begin{array}{c}\mathrm{HF} / \\
6-31 \mathrm{G}^{*}\end{array}$ & $\begin{array}{c}\mathrm{HF} / \\
6-311+\mathrm{G}^{*}\end{array}$ & $\begin{array}{l}\mathrm{MP} 2(\mathrm{fc}) / \\
6-31 \mathrm{G}^{*}\end{array}$ & $\begin{array}{l}\text { MP2(full)/ } \\
6-31 G^{*}\end{array}$ & $\begin{array}{l}\mathrm{MP} 2(\mathrm{fc}) / \\
\mathrm{cc}-\mathrm{pVDZ}\end{array}$ & $\begin{array}{c}\mathrm{MP} 2(\mathrm{fc}) / \\
\text { aug-cc-pV } \\
\mathrm{DZ}\end{array}$ \\
\hline 1 & 461.34206 & 461.41651 & 462.89749 & 462.96101 & 462.99265 & 463.06242 \\
\hline & 461.31309 & 461.40006 & 462.84107 & 462.90054 & 462.93955 & 463.01597 \\
\hline & 461.32665 & 461.41216 & 462.85531 & 462.91530 & 462.95276 & 463.02805 \\
\hline 26 & & & & & & \\
\hline
\end{tabular}

Table S4. Absolute energies ( $E$, -au), of octahedrane (1) and hydrocarbons 22 and 31 at various DFT levels of theory.

\begin{tabular}{lccccccc}
\hline Structure & $\begin{array}{c}\mathrm{B} 3 \mathrm{PW} 91 / \mathrm{a} \\
\mathrm{ug}-\mathrm{cc}-\mathrm{pVD} \\
\mathrm{Z}\end{array}$ & $\begin{array}{c}\mathrm{B} 3 \mathrm{PW} 91 / 6- \\
311+\mathrm{G}^{* *}\end{array}$ & $\begin{array}{c}\mathrm{B} 3 \mathrm{PW} 91 / 6 \\
-31+\mathrm{G}^{*}\end{array}$ & $\begin{array}{c}\mathrm{B} 3 \mathrm{PW} 91 / \mathrm{a} \\
\mathrm{ug}-\mathrm{cc}-\mathrm{pVD} \\
\mathrm{Z}\end{array}$ & $\begin{array}{c}\mathrm{B} 3 \mathrm{PW} 91 / 6 \\
-311+\mathrm{G}^{* *}\end{array}$ & $\begin{array}{c}\mathrm{B} 3 \mathrm{LYP} / \\
6-31+\mathrm{G}^{*}\end{array}$ & $\begin{array}{c}\mathrm{B} 3 \mathrm{LYP} / \\
6-31+\mathrm{G}^{*}\end{array}$ \\
\hline 464.33017 & 464.38491 & 464.291616 & 464.33017 & 464.38491 & 464.43709 & 464.43709 \\
& & & & & & & \\
\end{tabular}


Table S5. Absolute energies ( $E,-\mathrm{au})$, of octahedrane (1) and hydrocarbons 22 and $\mathbf{3 1}$ at various DFT levels of theory.

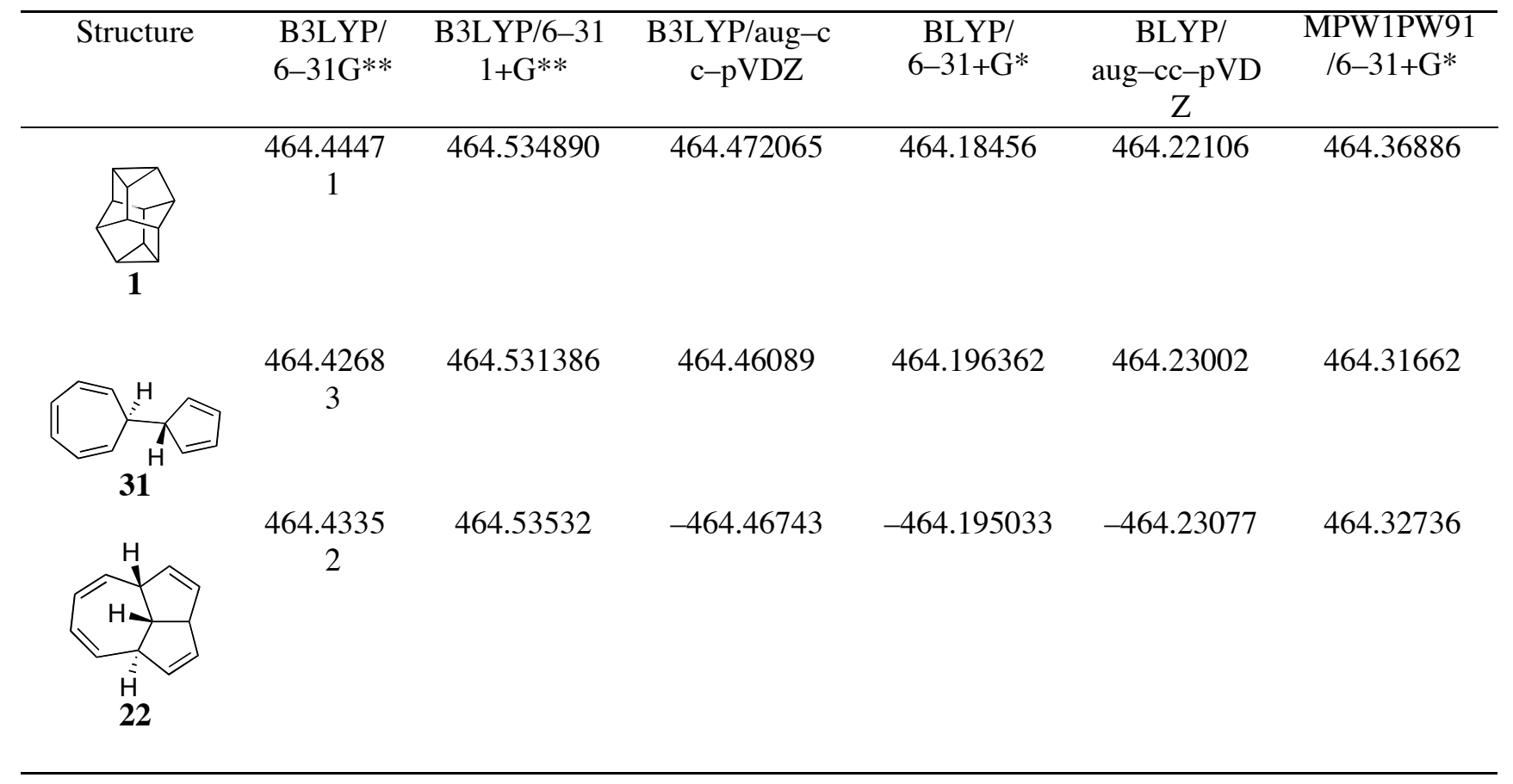

Table S6. Absolute energies ( $E,-\mathrm{au})$, of octahedrane (1) and hydrocarbons 22 and $\mathbf{3 1}$ at various DFT levels of theory.

\begin{tabular}{|c|c|c|c|c|}
\hline Structure & $\begin{array}{l}\text { G96LYP/ } \\
6-31+G^{*}\end{array}$ & $\begin{array}{c}\text { G96LYP/ } \\
\text { aug-cc-pVDZ }\end{array}$ & $\begin{array}{l}\text { BHandH/ } \\
6-31+\mathrm{G}^{*}\end{array}$ & $\begin{array}{c}\text { BHandHLYP/6-31+ } \\
\text { G* }^{*}\end{array}$ \\
\hline & 464.14779 & 464.18565 & 460.895876478 & 464.15850 \\
\hline & 464.15243 & 464.18758 & 460.81398 & 464.12946 \\
\hline & 464.15222 & 464.18943 & 460.83834 & 464.14102 \\
\hline & & & & \\
\hline
\end{tabular}


Table S7. Absolute CCSD(T) and QCISD(T) energies $(E,-\mathrm{au})$, of octahedrane (1) and hydrocarbons $\mathbf{2 2}$ and $\mathbf{3 1}$ at different $a b$ initio and DFT optimized geometries.

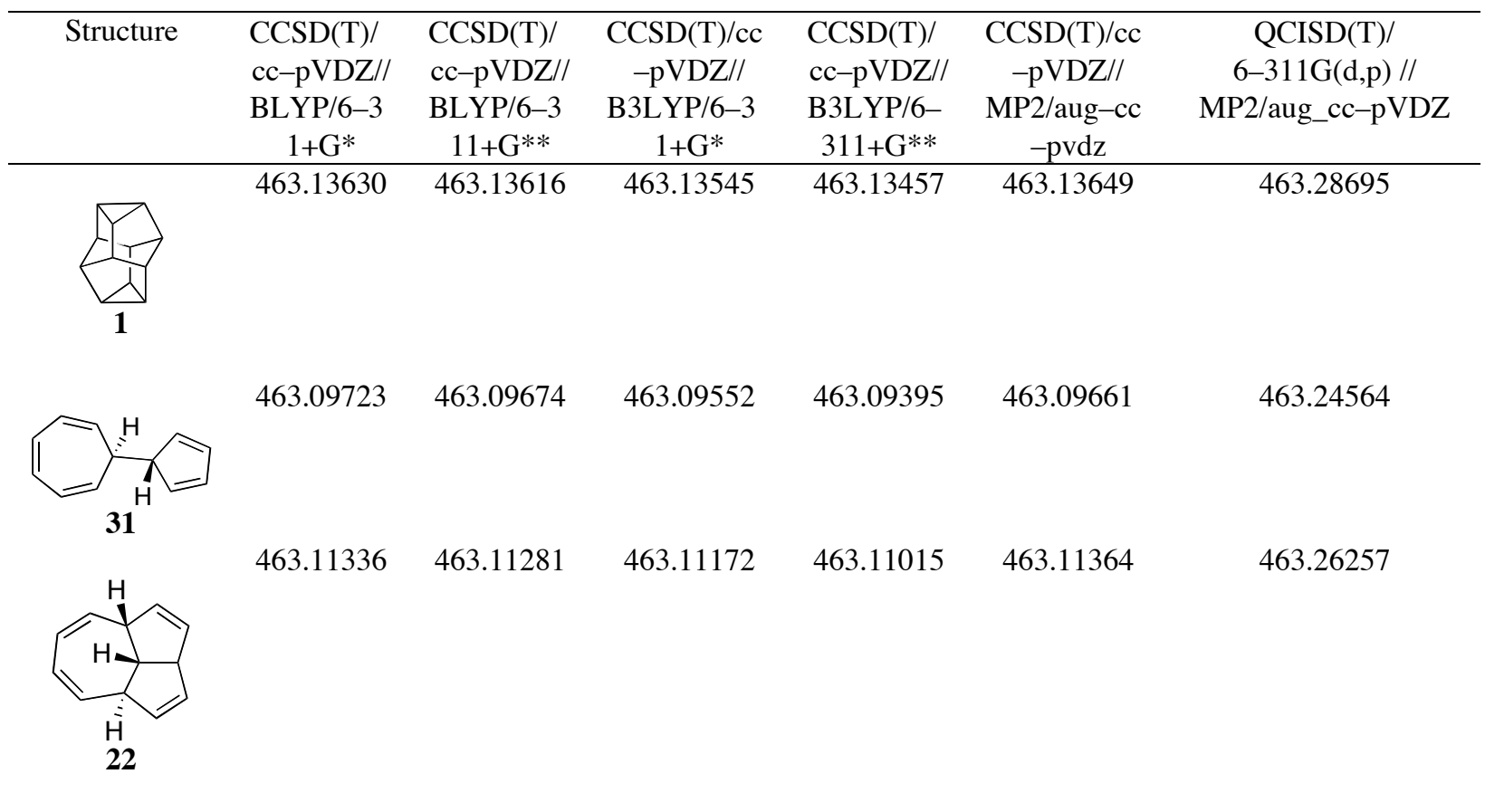

Table S8. Absolute and relative energies of model $\mathrm{C}_{6} \mathrm{H}_{6}$ hydrocarbons.

\begin{tabular}{|c|c|c|c|c|c|c|}
\hline Structure & $\begin{array}{c}\text { exp } \\
\Delta_{\mathrm{f}} \mathrm{H}^{\circ} \\
\text { gas } \\
\mathrm{kcal} / \\
\text { mol } \\
\text { (NIST) }\end{array}$ & $\begin{array}{c}\exp \\
\Delta \Delta_{\mathrm{f}} \mathrm{H}^{\circ} \\
\text { gas } \\
\mathrm{kcal} / \\
\mathrm{mol}\end{array}$ & $\begin{array}{c}\mathrm{B} 3 \mathrm{LYP} / 6-31 \mathrm{G}^{* *} \\
-\mathrm{au}\end{array}$ & $\begin{array}{c}\text { B3LYP/6-31G** } \\
\Delta \Delta_{\mathrm{f}} \mathrm{H}_{298} \\
\mathrm{kcal} / \\
\mathrm{mol}\end{array}$ & $\begin{array}{c}\mathrm{MP} 2 / 6-31 \mathrm{G}^{* *} \\
-\mathrm{au}\end{array}$ & $\begin{array}{c}\mathrm{MP} 2 / 6-31 \mathrm{G}^{* *} \\
\Delta \Delta_{\mathrm{f}} \mathrm{H}_{298} \\
\mathrm{kcal} / \\
\mathrm{mol}\end{array}$ \\
\hline & $\begin{array}{c}19.82 \pm \\
0.12\end{array}$ & 0.0 & $\begin{array}{l}232.25820(\mathrm{E}) \\
232.15759\left(\mathrm{E}_{0}\right) \\
232.15226\left(\mathrm{H}_{298}\right) \\
232.18506\left(\mathrm{G}_{298}\right)\end{array}$ & 0.0 & $\begin{array}{l}231.50539(\mathrm{E}) \\
231.40418\left(\mathrm{E}_{0}\right) \\
231.39866\left(\mathrm{H}_{298}\right) \\
231.43174\left(\mathrm{G}_{298}\right)\end{array}$ & 0.0 \\
\hline & 53.6 & 33.8 & $\begin{array}{l}232.20102(\mathrm{E}) \\
232.10282\left(\mathrm{E}_{0}\right) \\
232.09693\left(\mathrm{H}_{298}\right) \\
232.13079\left(\mathrm{G}_{298}\right)\end{array}$ & 34.7 & $\begin{array}{l}231.44623(\mathrm{E}) \\
231.34732\left(\mathrm{E}_{0}\right) \\
231.34130\left(\mathrm{H}_{298}\right) \\
231.37539\left(\mathrm{G}_{298}\right)\end{array}$ & 36.0 \\
\hline & 80.4 & 60.6 & $\begin{array}{l}232.15476(\mathrm{E}) \\
232.05848\left(\mathrm{E}_{0}\right) \\
232.05203\left(\mathrm{H}_{298}\right) \\
232.08638\left(\mathrm{G}_{298}\right)\end{array}$ & 62.9 & $\begin{array}{l}231.39389(\mathrm{E}) \\
231.29708\left(\mathrm{E}_{0}\right) \\
231.29042\left(\mathrm{H}_{298}\right) \\
231.32582\left(\mathrm{G}_{298}\right)\end{array}$ & 67.9 \\
\hline & 90.20 & 70.4 & $\begin{array}{l}232.14596(\mathrm{E}) \\
232.05084\left(\mathrm{E}_{0}\right) \\
232.04241\left(\mathrm{H}_{298}\right) \\
232.08348\left(\mathrm{G}_{298}\right)\end{array}$ & 68.9 & $\begin{array}{l}231.39252(\mathrm{E}) \\
231.29704\left(\mathrm{E}_{0}\right) \\
231.28791\left(\mathrm{H}_{298}\right) \\
231.33058\left(\mathrm{G}_{298}\right)\end{array}$ & 69.5 \\
\hline & $\begin{array}{c}94.6 \pm \\
2.9\end{array}$ & 74.8 & $\begin{array}{l}232.12308(\mathrm{E}) \\
232.02851\left(\mathrm{E}_{0}\right) \\
232.02147\left(\mathrm{H}_{298}\right) \\
232.05773\left(\mathrm{G}_{298}\right)\end{array}$ & 82.1 & $\begin{array}{l}231.35543(\mathrm{E}) \\
231.25966\left(\mathrm{E}_{0}\right) \\
231.25249\left(\mathrm{H}_{298}\right) \\
231.28903\left(\mathrm{G}_{298}\right)\end{array}$ & 91.7 \\
\hline
\end{tabular}


Table S9. Absolute and relative energies of model $\mathrm{C}_{6} \mathrm{H}_{8}$ hydrocarbons.

\begin{tabular}{|c|c|c|c|c|c|c|}
\hline Structure & $\begin{array}{c}\exp \\
\Delta_{\mathrm{f}} \mathrm{H}^{\circ} \\
\text { gas } \\
\mathrm{kcal} / \\
\mathrm{mol}\end{array}$ & $\begin{array}{c}\exp \\
\Delta \Delta_{\mathrm{f}} \mathrm{H}^{\circ} \\
\text { gas } \\
\mathrm{kcal} / \\
\mathrm{mol}\end{array}$ & $\begin{array}{c}\mathrm{B} 3 \mathrm{LYP} / 6-31 \mathrm{G}^{* *} \\
-\mathrm{au}\end{array}$ & $\begin{array}{c}\text { B3LYP/6-31G** } \\
\Delta \Delta_{\mathrm{f}} \mathrm{H}_{298} \\
\mathrm{kcal} / \mathrm{mol}\end{array}$ & $\begin{array}{c}\mathrm{MP} 2 / 6-31 \mathrm{G}^{* *} \\
-\mathrm{au}\end{array}$ & $\begin{array}{c}\text { MP2/6-31G** } \\
\Delta \Delta_{\mathrm{f}} \mathrm{H}_{298} \\
\mathrm{kcal} / \mathrm{mol}\end{array}$ \\
\hline & $\begin{array}{c}25.00 \pm \\
0.15\end{array}$ & 0.0 & $\begin{array}{l}233.43099(\mathrm{E}) \\
233.30848\left(\mathrm{E}_{0}\right) \\
233.30236\left(\mathrm{H}_{298}\right) \\
233.33674\left(\mathrm{G}_{298}\right)\end{array}$ & 0.0 & $\begin{array}{l}232.65780(\mathrm{E}) \\
232.53275\left(\mathrm{E}_{0}\right) \\
232.52667\left(\mathrm{H}_{298}\right) \\
232.56092\left(\mathrm{G}_{298}\right)\end{array}$ & 0.0 \\
\hline & 24.00 & 1.0 & $\begin{array}{l}233.43059(\mathrm{E}) \\
233.30836\left(\mathrm{E}_{0}\right) \\
233.30216\left(\mathrm{H}_{298}\right) \\
233.33685\left(\mathrm{G}_{298}\right)\end{array}$ & 0.1 & $\begin{array}{l}232.65545(\mathrm{E}) \\
232.53068\left(\mathrm{E}_{0}\right) \\
232.52436\left(\mathrm{H}_{298}\right) \\
232.55955\left(\mathrm{G}_{298}\right)\end{array}$ & 1.4 \\
\hline & 27.6 & 2.6 & $\begin{array}{l}233.43027(\mathrm{E}) \\
233.30881\left(\mathrm{E}_{0}\right) \\
233.30229\left(\mathrm{H}_{298}\right) \\
233.33807\left(\mathrm{G}_{298}\right)\end{array}$ & 0.0 & $\begin{array}{l}232.65526(\mathrm{E}) \\
232.53126\left(\mathrm{E}_{0}\right) \\
232.52480\left(\mathrm{H}_{298}\right) \\
232.56018\left(\mathrm{G}_{298}\right)\end{array}$ & 1.1 \\
\hline & 37.8 & 12.8 & $\begin{array}{l}233.40997(\mathrm{E}) \\
233.28748\left(\mathrm{E}_{0}\right) \\
233.28173\left(\mathrm{H}_{298}\right) \\
233.31524\left(\mathrm{G}_{298}\right)\end{array}$ & 12.9 & $\begin{array}{l}232.64442(\mathrm{E}) \\
232.51883\left(\mathrm{E}_{0}\right) \\
232.51315\left(\mathrm{H}_{298}\right) \\
232.54656\left(\mathrm{G}_{298}\right)\end{array}$ & 8.4 \\
\hline & 48.8 & 23.8 & $\begin{array}{l}233.39415(\mathrm{E}) \\
233.27470\left(\mathrm{E}_{0}\right) \\
233.26773\left(\mathrm{H}_{298}\right) \\
233.30430\left(\mathrm{G}_{298}\right)\end{array}$ & 21.7 & $\begin{array}{l}232.61488(\mathrm{E}) \\
232.49314\left(\mathrm{E}_{0}\right) \\
232.48607\left(\mathrm{H}_{298}\right) \\
232.52269\left(\mathrm{G}_{298}\right)\end{array}$ & 25.5 \\
\hline & 60.0 & 35.0 & $\begin{array}{l}233.37711(\mathrm{E}) \\
233.25418\left(\mathrm{E}_{0}\right) \\
233.24882\left(\mathrm{H}_{298}\right) \\
233.28152\left(\mathrm{G}_{298}\right)\end{array}$ & 33.6 & $\begin{array}{l}232.61630(\mathrm{E}) \\
232.49058\left(\mathrm{E}_{0}\right) \\
232.48529\left(\mathrm{H}_{298}\right) \\
232.51788\left(\mathrm{G}_{298}\right)\end{array}$ & 26.0 \\
\hline & 63.3 & 38.3 & $\begin{array}{l}233.38140(\mathrm{E}) \\
233.26377\left(\mathrm{E}_{0}\right) \\
233.25533\left(\mathrm{H}_{298}\right) \\
233.29500\left(\mathrm{G}_{298}\right)\end{array}$ & 29.5 & $\begin{array}{l}232.59284(\mathrm{E}) \\
232.47300\left(\mathrm{E}_{0}\right) \\
232.46435\left(\mathrm{H}_{298}\right) \\
232.50454\left(\mathrm{G}_{298}\right)\end{array}$ & 39.1 \\
\hline & 63.3 & 38.3 & $\begin{array}{l}233.38139(\mathrm{E}) \\
233.26376\left(\mathrm{E}_{0}\right) \\
233.25534\left(\mathrm{H}_{298}\right) \\
233.29508\left(\mathrm{G}_{298}\right)\end{array}$ & 29.5 & $\begin{array}{l}232.59286(\mathrm{E}) \\
232.47299\left(\mathrm{E}_{0}\right) \\
232.46438\left(\mathrm{H}_{298}\right) \\
232.50460\left(\mathrm{G}_{298}\right)\end{array}$ & 39.1 \\
\hline
\end{tabular}


Table S10. Absolute and relative energies of model $\mathrm{C}_{7} \mathrm{H}_{8}$ hydrocarbons.

\begin{tabular}{|c|c|c|c|c|c|c|}
\hline Structure & $\begin{array}{c}\exp \\
\Delta_{\mathrm{f}} \mathrm{H}^{\circ} \text { gas } \\
\mathrm{kcal} / \mathrm{mol}\end{array}$ & $\begin{array}{c}\exp \\
\Delta \Delta_{\mathrm{f}} \mathrm{H}^{\circ} \\
\text { gas } \\
\mathrm{kcal} / \\
\mathrm{mol}\end{array}$ & $\begin{array}{c}\mathrm{B} 3 \mathrm{LYP} / 6-31 \mathrm{G}^{* *} \\
-\mathrm{au}\end{array}$ & $\begin{array}{c}\text { B3LYP/6-31G** } \\
\Delta \Delta_{\mathrm{f}} \mathrm{H}_{298} \\
\mathrm{kcal} / \mathrm{mol}\end{array}$ & $\begin{array}{c}\mathrm{MP} 2 / 6-31 \mathrm{G}^{* *} \\
-\mathrm{au}\end{array}$ & $\begin{array}{c}\mathrm{MP} 2 / 6-31 \mathrm{G}^{* *} \\
\Delta \Delta_{\mathrm{f}} \mathrm{H}_{298} \\
\mathrm{kcal} / \mathrm{mol}\end{array}$ \\
\hline & $\begin{array}{c}11.95 \pm \\
0.15\end{array}$ & 0.0 & $\begin{array}{l}271.57878(\mathrm{E}) \\
271.45075\left(\mathrm{E}_{0}\right) \\
271.44359\left(\mathrm{H}_{298}\right) \\
271.48147\left(\mathrm{G}_{298}\right)\end{array}$ & 0.0 & $\begin{array}{l}270.69229(\mathrm{E}) \\
270.56270\left(\mathrm{E}_{0}\right) \\
270.55541\left(\mathrm{H}_{298}\right) \\
270.59301\left(\mathrm{G}_{298}\right)\end{array}$ & 0.0 \\
\hline & 46.99 & 35.0 & $\begin{array}{l}271.52288(\mathrm{E}) \\
271.39581\left(\mathrm{E}_{0}\right) \\
271.38876\left(\mathrm{H}_{298}\right) \\
271.42585\left(\mathrm{G}_{298}\right)\end{array}$ & 34.4 & $\begin{array}{l}270.63116(\mathrm{E}) \\
270.50196\left(\mathrm{E}_{0}\right) \\
270.49495\left(\mathrm{H}_{298}\right) \\
270.53163\left(\mathrm{G}_{298}\right)\end{array}$ & 37.9 \\
\hline & 44.2 & 32.2 & $\begin{array}{l}271.52424(\mathrm{E}) \\
271.39784\left(\mathrm{E}_{0}\right) \\
271.39041\left(\mathrm{H}_{298}\right) \\
271.42805\left(\mathrm{G}_{298}\right)\end{array}$ & 33.4 & $\begin{array}{l}270.63463(\mathrm{E}) \\
270.50670\left(\mathrm{E}_{0}\right) \\
270.49908\left(\mathrm{H}_{298}\right) \\
270.53712\left(\mathrm{G}_{298}\right)\end{array}$ & 35.3 \\
\hline & 44.6 & 32.6 & $\begin{array}{l}271.52196(\mathrm{E}) \\
271.39384\left(\mathrm{E}_{0}\right) \\
271.38715\left(\mathrm{H}_{298}\right) \\
271.42298\left(\mathrm{G}_{298}\right)\end{array}$ & 35.4 & $\begin{array}{l}270.63484(\mathrm{E}) \\
270.50457\left(\mathrm{E}_{0}\right) \\
270.49793\left(\mathrm{H}_{298}\right) \\
270.53361\left(\mathrm{G}_{298}\right)\end{array}$ & 36.1 \\
\hline & 56.8 & 44.8 & $\begin{array}{l}271.50301(\mathrm{E}) \\
271.37582\left(\mathrm{E}_{0}\right) \\
271.36906\left(\mathrm{H}_{298}\right) \\
271.40509\left(\mathrm{G}_{298}\right)\end{array}$ & 46.8 & $\begin{array}{l}270.62246(\mathrm{E}) \\
270.49274\left(\mathrm{E}_{0}\right) \\
270.48602\left(\mathrm{H}_{298}\right) \\
270.52199\left(\mathrm{G}_{298}\right)\end{array}$ & 43.5 \\
\hline & 58.4 & 46.4 & $\begin{array}{l}271.48975(\mathrm{E}) \\
271.36101\left(\mathrm{E}_{0}\right) \\
271.35520\left(\mathrm{H}_{298}\right) \\
271.38913\left(\mathrm{G}_{298}\right)\end{array}$ & 55.5 & $\begin{array}{l}270.61771(\mathrm{E}) \\
270.48649\left(\mathrm{E}_{0}\right) \\
270.48075\left(\mathrm{H}_{298}\right) \\
270.51457\left(\mathrm{G}_{298}\right)\end{array}$ & 46.8 \\
\hline & 63.2 & 51.2 & $\begin{array}{l}271.48730(\mathrm{E}) \\
271.35961\left(\mathrm{E}_{0}\right) \\
271.35322\left(\mathrm{H}_{298}\right) \\
271.38850\left(\mathrm{G}_{298}\right)\end{array}$ & 56.7 & $\begin{array}{l}270.60706(\mathrm{E}) \\
270.47685\left(\mathrm{E}_{0}\right) \\
270.47047\left(\mathrm{H}_{298}\right) \\
270.50577\left(\mathrm{G}_{298}\right)\end{array}$ & 53.3 \\
\hline & 80.4 & 68.4 & $\begin{array}{l}271.45756(\mathrm{E}) \\
271.32894\left(\mathrm{E}_{0}\right) \\
271.32341\left(\mathrm{H}_{298}\right) \\
271.35682\left(\mathrm{G}_{298}\right)\end{array}$ & 75.4 & $\begin{array}{l}270.58544(\mathrm{E}) \\
270.45372\left(\mathrm{E}_{0}\right) \\
270.44828\left(\mathrm{H}_{298}\right) \\
270.48155\left(\mathrm{G}_{298}\right)\end{array}$ & 67.2 \\
\hline & 88.4 & 76.4 & $\begin{array}{l}271.44375(\mathrm{E}) \\
271.31599\left(\mathrm{E}_{0}\right) \\
271.31014\left(\mathrm{H}_{298}\right) \\
271.34414\left(\mathrm{G}_{298}\right)\end{array}$ & 83.7 & $\begin{array}{l}270.57330(\mathrm{E}) \\
270.44237\left(\mathrm{E}_{0}\right) \\
270.43663\left(\mathrm{H}_{298}\right) \\
270.47046\left(\mathrm{G}_{298}\right)\end{array}$ & 74.5 \\
\hline & 91.76 & 79.8 & $\begin{array}{l}271.44310(\mathrm{E}) \\
271.31569\left(\mathrm{E}_{0}\right) \\
271.30952\left(\mathrm{H}_{298}\right) \\
271.34422\left(\mathrm{G}_{298}\right)\end{array}$ & 84.1 & $\begin{array}{l}270.56729(\mathrm{E}) \\
270.43703\left(\mathrm{E}_{0}\right) \\
270.43092\left(\mathrm{H}_{298}\right) \\
270.46554\left(\mathrm{G}_{298}\right)\end{array}$ & 78.1 \\
\hline
\end{tabular}


Table S11. Absolute and relative energies of model $\mathrm{C}_{8} \mathrm{H}_{10}$ hydrocarbons.

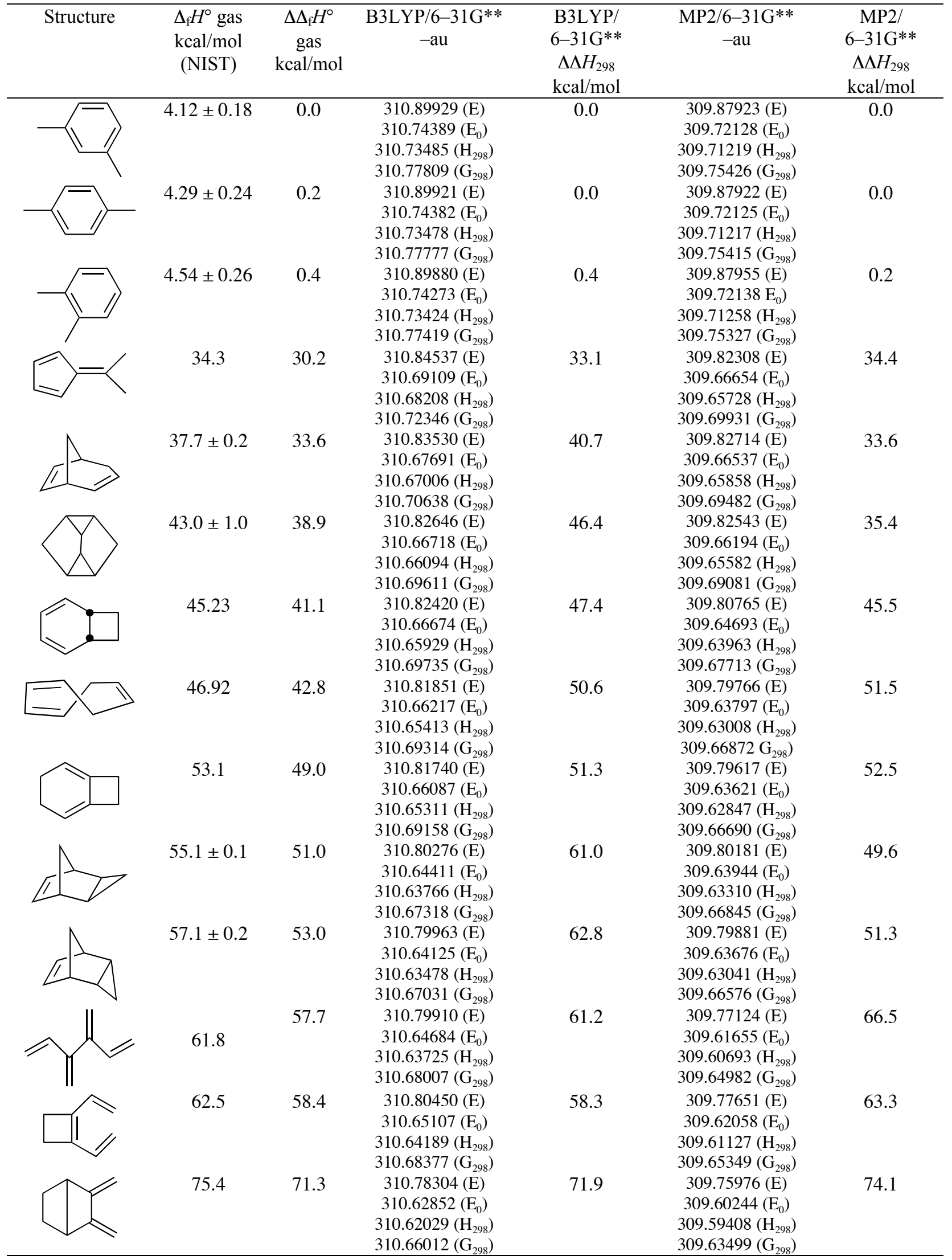


Table S12. Absolute and relative energies of model $\mathrm{C}_{9} \mathrm{H}_{12}$ hydrocarbons.

\begin{tabular}{|c|c|c|c|c|c|c|}
\hline Structure & $\begin{array}{l}\Delta_{\mathrm{f}} H^{\circ} \text { gas } \\
\mathrm{kcal} / \mathrm{mol}\end{array}$ & $\begin{array}{l}\Delta \Delta_{\mathrm{f}} H^{\circ} \text { gas } \\
\mathrm{kcal} / \mathrm{mol}\end{array}$ & $\begin{array}{c}\mathrm{B} 3 \mathrm{LYP} / 6-31 \mathrm{G}^{* *} \\
-\mathrm{au}\end{array}$ & $\begin{array}{c}\mathrm{B} 3 \mathrm{LYP} / 6-31 \mathrm{G} \\
* * \\
\Delta \Delta H_{298} \\
\mathrm{kcal} / \mathrm{mol}\end{array}$ & $\begin{array}{c}\mathrm{MP} 2 / 6-31 \mathrm{G}^{* *} \\
-\mathrm{au}\end{array}$ & $\begin{array}{c}\mathrm{MP} 2 / 6-31 \mathrm{G}^{* *} \\
\Delta \Delta H_{298} \\
\mathrm{kcal} / \mathrm{mol}\end{array}$ \\
\hline & $\begin{array}{l}-3.33 \pm \\
0.27\end{array}$ & 0.0 & $\begin{array}{c}350.21924(\mathrm{E}) \\
350.03578\left(\mathrm{E}_{0}\right) \\
350.02542\left(\mathrm{H}_{298}\right) \\
350.07024\left(\mathrm{G}_{298}\right)\end{array}$ & 0.0 & $\begin{array}{c}349.06655(\mathrm{E}) \\
348.88002\left(\mathrm{E}_{0}\right) \\
348.86939\left(\mathrm{H}_{298}\right) \\
348.91456\left(\mathrm{G}_{298}\right)\end{array}$ & 0.0 \\
\hline & $\begin{array}{c}26.09 \pm \\
0.31\end{array}$ & 29.4 & $\begin{array}{c}350.16030(\mathrm{E}) \\
349.97347\left(\mathrm{E}_{0}\right) \\
349.96503\left(\mathrm{H}_{298}\right) \\
350.00581\left(\mathrm{G}_{298}\right)\end{array}$ & 37.9 & $\begin{array}{c}349.01382(\mathrm{E}) \\
348.82285\left(\mathrm{E}_{0}\right) \\
348.81453\left(\mathrm{H}_{298}\right) \\
348.85502\left(\mathrm{G}_{298}\right)\end{array}$ & 34.3 \\
\hline & $\begin{array}{c}35.07 \pm \\
0.21\end{array}$ & 38.4 & $\begin{array}{c}350.14410(\mathrm{E}) \\
349.95865\left(\mathrm{E}_{0}\right) \\
349.94989\left(\mathrm{H}_{298}\right) \\
349.99081\left(\mathrm{G}_{298}\right)\end{array}$ & 47.4 & $\begin{array}{c}349.00150(\mathrm{E}) \\
348.81189\left(\mathrm{E}_{0}\right) \\
348.80319\left(\mathrm{H}_{298}\right) \\
348.84409\left(\mathrm{G}_{298}\right)\end{array}$ & 41.5 \\
\hline & $38.0 \pm 0.2$ & 41.3 & $\begin{array}{c}350.13704(\mathrm{E}) \\
349.95093\left(\mathrm{E}_{0}\right) \\
349.94247\left(\mathrm{H}_{298}\right) \\
349.98287\left(\mathrm{G}_{298}\right)\end{array}$ & 52.1 & $\begin{array}{c}348.99640(\mathrm{E}) \\
348.80638\left(\mathrm{E}_{0}\right) \\
348.79802\left(\mathrm{H}_{298}\right) \\
348.83829\left(\mathrm{G}_{298}\right)\end{array}$ & 44.8 \\
\hline & 56.5 & 59.8 & $\begin{array}{c}350.11321(\mathrm{E}) \\
349.93010\left(\mathrm{E}_{0}\right) \\
349.92062\left(\mathrm{H}_{298}\right) \\
349.96263\left(\mathrm{G}_{298}\right)\end{array}$ & 65.8 & $\begin{array}{c}348.96552(\mathrm{E}) \\
348.77823\left(\mathrm{E}_{0}\right) \\
348.76883\left(\mathrm{H}_{298}\right) \\
348.81076\left(\mathrm{G}_{298}\right)\end{array}$ & 63.1 \\
\hline & $101.2 \pm 1.2$ & 104.5 & $\begin{array}{c}350.04226(\mathrm{E}) \\
349.86011\left(\mathrm{E}_{0}\right) \\
349.85048\left(\mathrm{H}_{298}\right) \\
349.89319\left(\mathrm{G}_{298}\right)\end{array}$ & 109.8 & $\begin{array}{c}348.89697(\mathrm{E}) \\
348.70932\left(\mathrm{E}_{0}\right) \\
348.69989\left(\mathrm{H}_{298}\right) \\
348.74226\left(\mathrm{G}_{298}\right)\end{array}$ & 106.4 \\
\hline & 102.7 & 106.0 & $\begin{array}{c}350.04215(\mathrm{E}) \\
349.86000\left(\mathrm{E}_{0}\right) \\
349.85047\left(\mathrm{H}_{298}\right) \\
349.89311\left(\mathrm{G}_{298}\right)\end{array}$ & 109.8 & $\begin{array}{c}348.89622(\mathrm{E}) \\
348.70851\left(\mathrm{E}_{0}\right) \\
348.69919\left(\mathrm{H}_{298}\right) \\
348.74145\left(\mathrm{G}_{298}\right)\end{array}$ & 106.8 \\
\hline
\end{tabular}


Table S13. Absolute and relative energies of model $\mathrm{C}_{10} \mathrm{H}_{10}$ hydrocarbons.

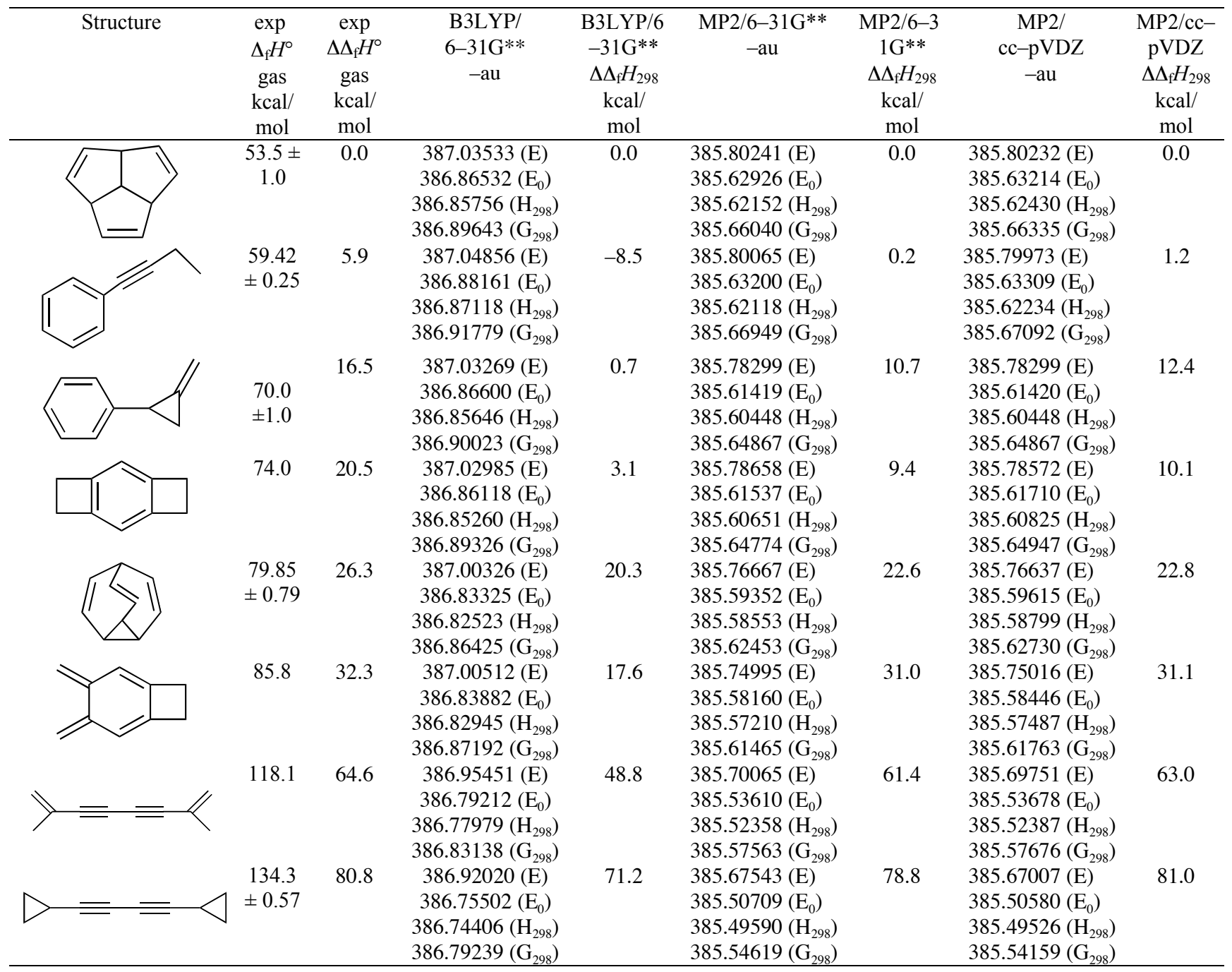


Table S14. Absolute and relative energies of model $\mathrm{C}_{10} \mathrm{H}_{14}$ hydrocarbons.

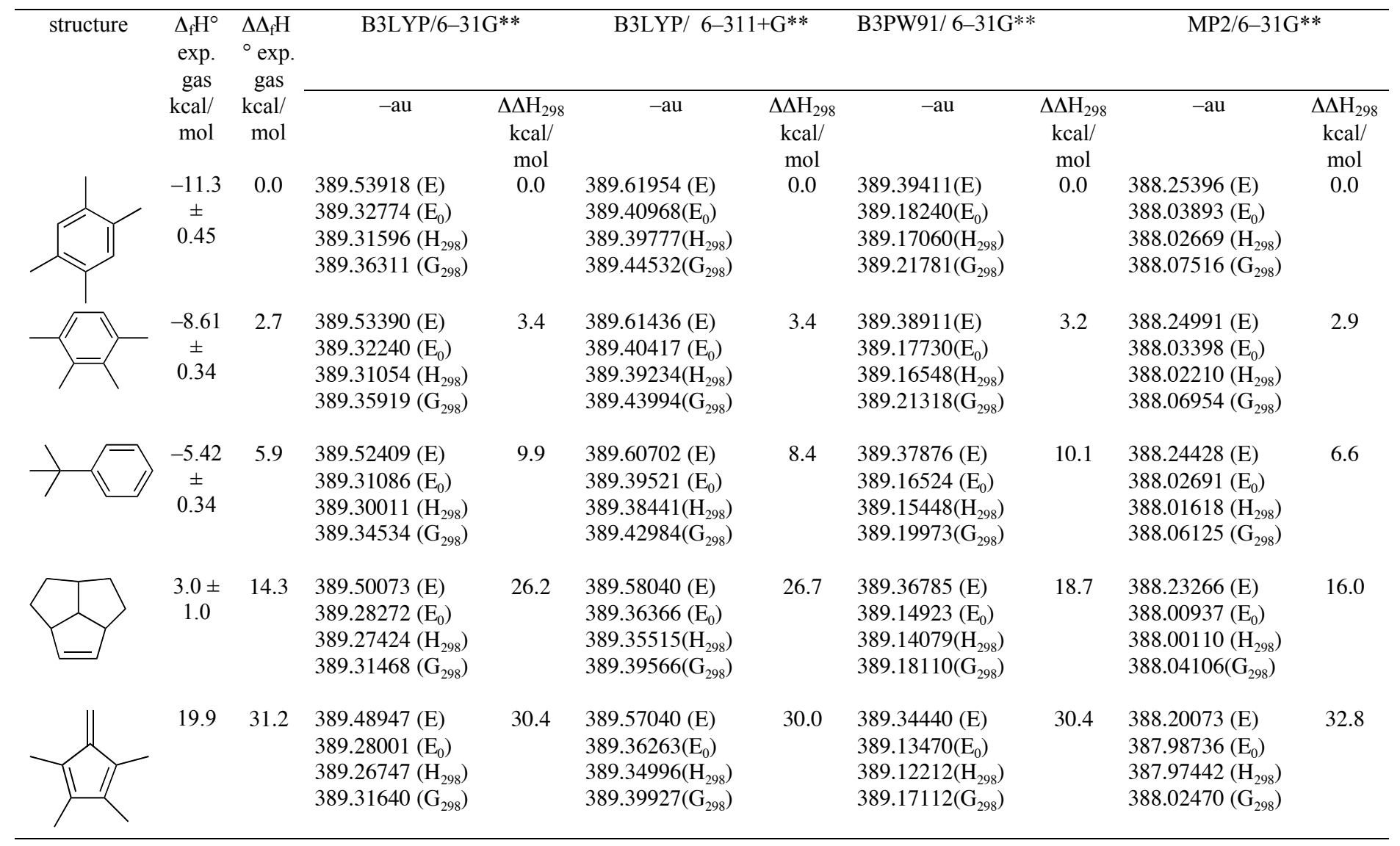


Table S15. Absolute and relative energies of model $\mathrm{C}_{12} \mathrm{H}_{16}$ hydrocarbons.

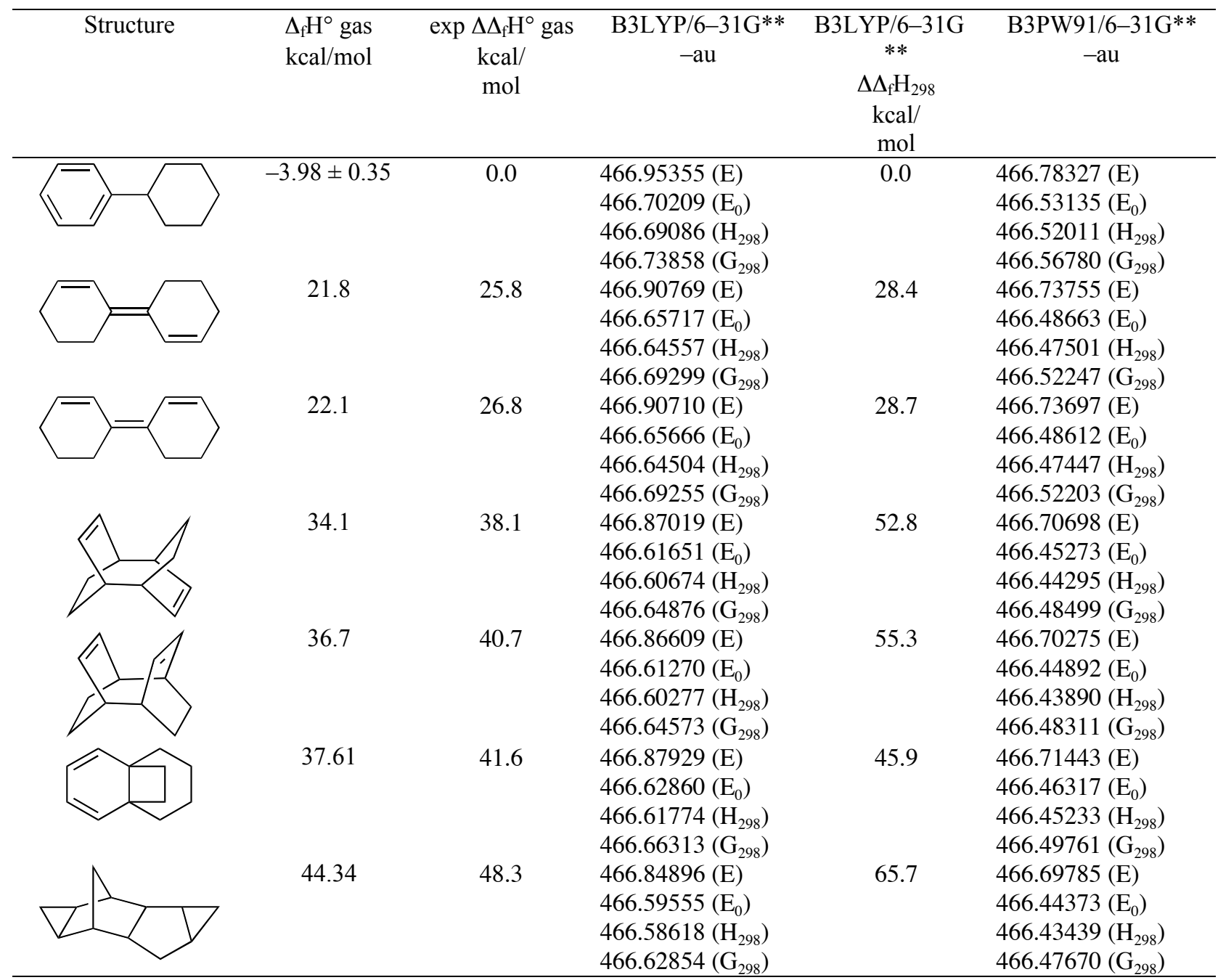

